\title{
Management of febrile neutropenia in the United Kingdom: time for a national trial?
}

\author{
H Innes', L Billingham², C Gaunt ${ }^{2}$, N Steven ${ }^{2}$ and E Marshall ${ }^{*, 1}$ \\ 'Clatterbridge Centre for Oncology, Bebington, Wirral, Merseyside CH63 4JY, UK; ${ }^{2}$ Cancer Research UK Institute for Cancer Studies, University \\ of Birmingham, Birmingham, UK
}

Recent advances in febrile neutropenia (FN) have highlighted the value of risk stratification and the evolving role of oral antibiotics with early hospital discharge in low-risk patients. The aim of this study was to survey whether these advances have been translated into routine clinical practice in the UK. Questionnaires were sent to cancer clinicians across the UK to determine clinicians' routine management of FN, including use of risk stratification, antibiotic regimen and criteria for hospital discharge. In all, 128 clinicians responded, representing 50 cancer departments (83\%). Only 38\% of respondents stratify patients according to risk and with substantial variation in the criteria defining 'low-risk'. Furthermore, only $22 \%$ of clinicians use oral antibiotics as first-line treatment in any patients with $\mathrm{FN}$, but this was significantly greater among clinicians who do compared to those who do not stratify patients by risk, 5 I vs $4 \%(P<0.000$ I). These findings suggest a slow and/or cautious introduction of newer strategies for the management of low-risk FN in the UK. However, $84 \%$ of respondents confirmed their willingness to participate in a trial of oral antibiotics combined with early discharge in low-risk FN.

British Journal of Cancer (2005) 93, 1324- 1328. doi:I0.1038/sj.bjc.6602872 www.bjcancer.com

(c) 2005 Cancer Research UK

Keywords: febrile neutropenia; low-risk; oral antibiotics; UK practice

For more than 30 years the standard management for all patients developing febrile episodes while neutropenic has been in-patient treatment with broad spectrum intravenous (i.v.) antibiotics (Hughes et al, 1990; Bodey et al, 1997; Hughes et al, 1997). However, recent evidence has prompted a re-assessment of febrile neutropenia (FN), with increasing realisation that such intensive treatment may not be necessary or appropriate for all patients. This viewpoint has stemmed from the recognition that FN is not a single entity but rather represents a spectrum of severity. Only a small proportion of patients with FN will develop serious medical complications ( $16 \%$ overall, $12 \%$ of patients with solid tumours/ lymphomas and $18 \%$ of patients with haematological malignancies) and fewer than $5 \%$ will die as a result of the episode $(4.8 \%$; Klastersky et al, 2000). Thus a majority of episodes of FN may be described as being 'low-risk'. Several groups of investigators have independently developed prognostic indices in FN in an attempt to identify criteria by which to define 'low-risk' (Rubin et al, 1988; Talcott et al, 1992; Viscoli et al, 1994; Elting et al, 1997; Talcott et al, 1998). More recently these groups have come together in an international collaboration with the publication of the Multinational Association for Supportive Care in Cancer (MASCC) 'risk index' (Klastersky et al, 2000). In this study more than 40 possible risk factors for the development of significant medical complications at presentation of FN were examined in an initial derivation set of 756 patients. From this, logistic regression analysis was used to derive a model for the risk of development of such

*Correspondence: Dr E Marshall; E-mail ernie.marshall@ccotrust.nhs.uk Received I July 2005; revised 12 October 2005; accepted 17 October 2005 complications, which was subsequently tested in a further validation set of patients. The resulting risk index consists of seven weighted clinical factors (Table 1). The value of this scoring system in the prediction of the development of serious complications has subsequently been validated in both the single-centre (Uys et al, 2004) and multicentre settings (Paesmans et al, 2003).

The aim of defining low-risk neutropenia in this way has been to identify episodes of $\mathrm{FN}$, which may be amenable to newer approaches to treatment involving less intensive, more convenient treatment, which may in turn be expected to have an impact on patients' quality of life. Such treatment strategies may also have a potentially significant impact on health service resource utilisation.

Most research effort has focussed on the role of oral antibiotics. Two large multinational prospective randomised controlled trials (Freifeld et al, 1999; Kern et al, 1999) have demonstrated equivalence in terms of both efficacy and safety for oral antibiotics compared to standard parenteral regimens for patients with lowrisk FN when delivered in the in-patient setting. These findings have led to the inclusion of combination oral antibiotics as a standard option for treatment of low-risk FN in the most recent Infectious Diseases Society of America guidelines for the use of antimicrobial agents in neutropenic patients with cancer (Hughes et al, 2002). The case for oral antibiotics has been further strengthened by a recent meta-analysis (Vidal et al, 2004) of 15 trials comparing oral and i.v. treatment, which found no difference in failure rates or mortality between the two interventions.

The feasibility of combining an oral antibiotic regimen with early hospital discharge has been reported in a UK population in a randomised single-centre trial (Innes et al, 2003) which compared this approach to standard in-patient i.v. treatment. In this study, 
Table I MASCC Risk index

\begin{tabular}{lr}
\hline Characteristic & Scor \\
\hline Burden of illness & \\
No or mild symptoms ${ }^{\text {a }}$ & 5 \\
Moderate symptoms $^{a}$ & 3 \\
No hypotension & 5 \\
No chronic obstructive pulmonary disease & 4 \\
Solid tumour/lymphoma or no previous fungal infection & 4 \\
No dehydration & 3 \\
Outpatient status at onset of fever & 3 \\
Age <60 years & 2
\end{tabular}

apoints attributable to burden of illness are not cumulative. The maximum theoretical score is therefore 26 . The authors used a threshold of $\geqslant 21$ points to define 'low-risk'.

not only were the success rates of the i.v. and oral arms comparable, but importantly the policy of oral antibiotics with early discharge reduced median in-patient stay to only 2 days. In turn this was associated with substantial savings both in financial terms and in the amount of nursing care required. Although a long-term aim, there is presently little randomised data to support the management of low-risk FN in the outpatient setting.

The evolving management of low-risk FN on an international level led us to investigate the current treatment policy of low-risk FN in oncology departments across the UK. In particular, we were interested to determine:

(i) Whether UK clinicians are assessing the likelihood of patients developing complications related to $\mathrm{FN}$ and if so, whether they are using the MASCC index or other means.

(ii) Whether UK clinicians are using oral antibiotics as first-line treatment in any patients with FN.

(iii) What criteria UK clinicians are using to determine patients' suitability for hospital discharge and whether they have policies for early hospital discharge.

\section{METHODS}

A database of clinicians registered to a UK national trial of prophylactic antibiotics (Cullen et al, 2005) was used to identify 249 consultant oncologists and haematologists from 60 centres across the UK with an interest in the antibiotic management of FN. Questionnaires were sent to these identified clinicians in September 2003 with a covering letter explaining its aims and rationale, a stamped addressed envelope and additional questionnaires to distribute to other relevant colleagues.

\section{The questionnaire}

The questionnaire was designed to be succinct (eight questions covering one sheet of paper) and easy to answer consisting of mainly tick-box responses. The name and hospital of the clinician was preprinted onto the questionnaire. Clinicians were initially asked whether they manage patients with FN. If not they were asked to return the survey uncompleted, but otherwise to proceed to answer the remaining questions. The questions related to the three key areas specified below and clinicians were invited to also give further comments.

Stratification and treatment of low-risk FN Clinicians were asked whether they routinely stratify patients with FN and, if so, to explain the criteria by which they defined 'low-risk'. They were then asked about their standard first-line antibiotic treatment for non-penicillin-allergic low-risk patients with FN. They were asked to specify whether they use an i.v. antibiotic regimen until resolution, an i.v. regimen initially followed by 'step-down' oral antibiotics or oral antibiotics from the outset and for the details of the regimen used. There was also an additional question asking about the timing of antibiotic discontinuation.

Criteria for hospital discharge Clinicians were asked for the criteria they use for hospital discharge for patients recovering from febrile netropenia. They were asked whether they take into account temperature, neutrophil count, a combination of both or other criteria. If temperature was used as a criterion for discharge clinicians were asked to further define the absolute value and duration of the patient's defervescence, which would be deemed appropriate for discharge. Similarly, clinicians who specified neutrophil count as a criterion were asked for the absolute neutrophil count which would be deemed appropriate for discharge or alternatively whether they used a rising neutrophil count, irrespective of the absolute value as their preferred criterion.

Trial participation Finally, clinicians were asked whether they would be willing to participate in a trial for low-risk FN using oral antibiotics in conjunction with early hospital discharge.

\section{Statistical analysis}

The responses of clinicians to the questionnaire are reported descriptively. Type of antibiotic treatment and criteria for hospital discharge are compared between those who do or do not stratify patients by risk using continuity-adjusted $\chi^{2}$ tests.

\section{RESULTS}

Of the 249 questionnaires sent out to clinicians from 60 cancer departments across the UK, replies were received from 114 (47.4\%) clinicians who manage patients with FN. A further 14 replies were received from clinicians who were not included in the original mailshot but who had been passed copies of the questionnaire by other recipients. Thus a total of 128 responding clinicians (94 clinical/medical oncologists; 32 haematologists and two others) who manage FN responded. These represent 50 departments across the UK with a median of two clinicians from each centre (range 1-7).

\section{Stratification of FN}

Of these 128 clinicians, 79 (62\%) do not stratify patients with FN into low- and high-risk categories (Figure 1). Of the $49(38 \%)$ who do stratify, three indicated that this was on an occasional or informal basis. Of the 50 oncology/haematology departments represented, $28(56 \%)$ had at least one clinician who stratifies patients by risk and $22(44 \%)$ had no responding clinician who stratifies such episodes by risk. Within the centres represented there was a lack of consistency regarding risk stratification; of the 29 centres from which two or more clinicians responded, there were only five centres from which all responding clinicians stratify by risk.

Of the 49 clinicians who stratify by risk, 43 gave the criteria by which they define 'low-risk', three stated that there were no formal criteria and three gave no further information. The criteria varied substantially, with the majority using a combination of factors including patients' symptoms and signs, absolute neutrophil count and expected duration of neutropenia, nature of the underlying malignancy, previous episodes of neutropenia and age (Table 2). However, very few clinicians appeared to be systematically using a single published definition of low-risk but rather to be using factors derived from more than one study. In addition, some clinicians gave more general and subjective definitions, for 


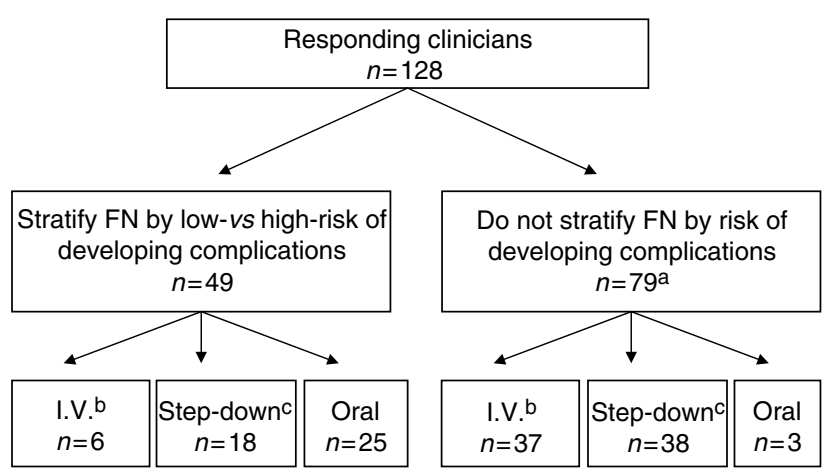

aOne respondent did not specify which antibiotic regimen was used. bl.V. regimen until resolution.

cl.V. antibiotics followed by change to oral regimen after unspecified period.

Figure I Summary of clinicians' antibiotic treatment of low-risk FN.

Table 2 Criteria used to define 'low-risk'
Factor used to stratify risk of FN

Clinical findings

Absolute neutrophil count

Anticipated duration of neutropenia

Patients' symptoms or performance status

Underlying malignancy (nonhaematological

or nonacute leukaemia)

Site of infection

Comorbidities

Age

Chemotherapy regimen

Previous fungal infection in haematological malignancy

Outpatient at presentation

Previous episodes of febrile neutropenia

'Controlled' cancer
No of clinicians using this factor

example, 'no sepsis', 'well' etc. Responding clinicians from only one centre (the author's) were routinely incorporating the MASCC risk-index into clinical practice. Clinicians who were using oral antibiotics for low-risk patients also gave patients' ability to tolerate oral medication, for example, not vomiting, able to tolerate oral medication, no severe mucositis as additional criteria for suitability for such treatment. In addition, clinicians who had a policy of early discharge also included additional criteria for their suitability for example, having a carer at home, proximity to hospital, availability of transport, together with subjective assessment of patients, for example, 'compliant patient', 'sensible patient'.

\section{Antibiotic regimens}

Overall $43(34 \%)$ clinicians use an i.v. antibiotic regimen until resolution as their standard first-line treatment for non-penicillin allergic patients with 'low-risk' FN, 56 (44\%) use i.v. antibiotics followed by oral antibiotics (a 'step-down' regimen) and 28 (22\%) use an oral antibiotic regimen from the outset in at least some of their patients (Figure 1). Of the 49 clinicians who stratify patients, $25(51 \%)$ use oral regimens from the outset, in marked contrast to the 79 clinicians not stratifying by risk of whom only three $(4 \%)$ used oral regimens $(P<0.0001)$. The range of different antibiotics regimens utilised is shown in Table 3.
Table 3 Antibiotic regimens used

\begin{tabular}{lc}
\hline Antibiotic regimen used & No. (\% of known) clinicians using \\
\hline Oral antibiotic regimens $(n=28)$ & $18(69)$ \\
Ciprofloxacin+co-amoxiclav & $8(31)$ \\
Ciprofloxacin & 2 \\
Not specified & \\
'Step-down' antibiotic regimens (intravenous $\rightarrow$ oral; $n=56)$ & $40(82)$ \\
Dual therapy $\rightarrow$ oral antibiotics & $9(18)$ \\
Monotherapy $\rightarrow$ oral antibiotics & 7 \\
Not specified & \\
Intravenous antibiotic regimens ( $n=43)$ & $25(68)$ \\
Dual therapy & $12(32)$ \\
Monotherapy & 6 \\
Not specified & \\
\hline
\end{tabular}

\section{Criteria for hospital discharge}

The criteria used by respondents for hospital discharge are shown in Table 4 . The majority $(n=91 ; 71 \%)$ used a combination of temperature and neutrophil count as criteria for hospital discharge (including four who stated that just one criteria is used in certain situations and one whose criteria also included five days i.v. antibiotics). Of the remaining respondents, 33 (26\%) used only the patient's temperature, two (2\%) used only the neutrophil count, one specified that discharge was at the clinician's discretion and one did not specify. Those who stratify patients according to risk were more likely to utilise fever lysis alone, irrespective of neutrophil count: 17 of the $49(35 \%)$ clinicians who do stratify by risk use temperature only compared with 16 of the $79(20 \%)$ nonstratifying clinicians although this did not reach statistical significance $(P=0.11)$. Of the 123 clinicians who specified temperature criteria for discharge, almost half $(n=60 ; 49 \%)$ specified $\leqslant 37^{\circ} \mathrm{C}$ and half $(n=60 ; 49 \%)$ specified $\leqslant 37.5^{\circ} \mathrm{C}$, with $24 \mathrm{~h}$ the most popular duration $(n=87 ; 71 \%)$. Of the 93 clinicians who gave patient's neutrophil count as part of their criteria for discharge approximately a third $(n=32 ; 34 \%)$ use a rising neutrophil count irrespective of the absolute value, while the remainder $(n=61 ; 66 \%)$ have a fixed absolute neutrophil count which they regard as being suitable for discharge.

\section{Duration of antibiotic treatment}

The majority of clinicians $(n=79 ; 62 \%)$ stated that in low-risk patients antibiotics were discontinued after a minimum number days following lysis of fever with 1-2 days specified by 14 clinicians, $2-5$ days by 54 and $5-7$ days by 11 . A further $27(21 \%)$ discontinue antibiotics at (or $24 \mathrm{~h}$ before; $n=1$ ) discharge. Nine clinicians $(7 \%)$ discontinue after a fixed duration from onset of fever with number of days ranging from 5 to 10 . The criteria for the remaining clinicians related to neutrophil recovery $(n=4)$ or white blood count $(n=1)$ and eight not specifying.

\section{Clinicians' comments and trial participation}

When asked for their further comments, clinicians from three centres commented that they manage some patients with low-risk FN as outpatients and a further five that they have early discharge policies in place. A number of clinicians also made comments regarding practical difficulties in the assessment and management of low-risk FN. In particular, several respondents cited difficulties with the ability of junior staff to assess risk, for example 'junior staff play it safe'; '(difficulty in determining whether) the nadir is passed or coming'. One clinician commented that 'in practice very 
Table 4 Criteria used for patient discharge. (a) Temperature and duration for those who use temperature criterion and (b) Neutrophil count criteria for those who use it

\begin{tabular}{|c|c|c|c|c|c|c|}
\hline & I reading & 2 readings $4 h$ apart & 3 readings $4 \mathrm{~h}$ apart & $24 \mathrm{~h}$ & Other $^{\mathbf{a}}$ & Total \\
\hline \multicolumn{7}{|l|}{ (a) } \\
\hline$\leqslant 37^{\circ} \mathrm{C}$ & 0 & 8 & I & 44 & 7 & 60 \\
\hline$\leqslant 37.5^{\circ} \mathrm{C}$ & 2 & 10 & 3 & 41 & 4 & 60 \\
\hline$\leqslant 37.9^{\circ} \mathrm{C}$ & 0 & । & 0 & 2 & 0 & 3 \\
\hline Total & 2 & 19 & 4 & 87 & 11 & $123^{b}$ \\
\hline \multicolumn{7}{|l|}{ (b) } \\
\hline & $\begin{array}{l}\text { Neutrophil } \\
\text { count only }\end{array}$ & $\begin{array}{l}\text { Temperature and } \\
\text { neutrophil count }\end{array}$ & Other & Total & & \\
\hline$\geqslant 0.5 \times 10^{9} / 1$ & 0 & 36 (and rising in I) & 0 & $36(39 \%)$ & & \\
\hline$\geqslant 1 \times 10^{9} / 1$ & I & 18 (and rising in 3 ) & 3 & $22(24 \%)$ & & \\
\hline Rising neurophil count irrespective of value & I & 29 & 2 & $32(34 \%)$ & & \\
\hline Other ${ }^{c}$ & 0 & 3 & 0 & $3(3 \%)$ & & \\
\hline Total & 2 & 86 & $5^{d}$ & 93 & & \\
\hline
\end{tabular}

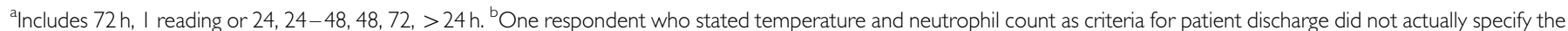
criteria. 'Includes $0.8 \times 10^{9} / \mathrm{l}, \geqslant 0.3 \times 10^{9} / \mathrm{l}, \geqslant 0.2 \times 10^{9} / \mathrm{l}$ and rising. ${ }^{\mathrm{d}}$ Two of the clinicians who specified 'other' criteria did not use neutrophil count.

rarely do patients get into low-risk category'. However, respondents were generally very favourable about the concept of early discharge for patients with low-risk FN, one clinician commenting that 'we have real pressure on beds, anything reducing in-patient stay would be of interest'. Of the 128 respondents, $108(84 \%)$ said that they would be willing to participate in a trial for low-risk FN of oral antibiotics combined with early hospital discharge.

\section{DISCUSSION}

This study is the first to survey the UK management of FN, an important complication of anticancer chemotherapy. Clinicians from 50 of the 60 cancer departments surveyed (83\%) responded, giving broad representation of current treatment across the UK. The findings suggest substantial variation in the management of FN and a slow and/or cautious introduction of recent evidence in this area.

Fewer than half of the responding clinicians stratify patients with FN according to their likelihood of developing complications. Moreover, of those who do stratify, the definition of 'low-risk' varies greatly, with clinicians using different combinations of a variety of published and unpublished, objective and subjective criteria. Clinicians from only one centre (the author's) were using the well-validated MASCC scoring system. Moreover, only a quarter $(22 \%)$ of respondents use oral antibiotics from the outset in the management of any of their patients with FN. This is despite the increasing evidence base, which demonstrates equivalence in terms of success rates and safety for oral $v s$ i.v. regimens in lowrisk FN (Freifeld et al, 1999; Kern et al, 1999; Innes et al, 2003), the anticipated benefits of quality of life to patients and the potential significant savings in utilisation of resources. Furthermore, the study findings represent the practice of clinical trialists in the UK in the field of FN. It may be expected that as such they are more likely to have incorporated recent study findings into their clinical practice. Therefore, this study may overestimate the use of oral antibiotics in the routine management of FN in the UK. The reasons for not implementing such policies have not been explored in the current survey, but it is possible that some clinicians feel that the level of staffing available is inadequate to safely implement the close monitoring required for such policies.

Interestingly, clinicians and centres that do stratify and thus identify low-risk patients were more likely to have introduced oral antibiotics into their management of FN than those who do not stratify. Thus acknowledgement of the concept of 'low-risk' is accompanied by recognition that a 'one size fits all' management strategy is not necessarily the most appropriate treatment of FN. Similarly, clinicians who stratified by risk were more likely to use only patients' temperature rather than temperature together with neutrophil count as criteria for determining discharge from hospital than those who do not stratify by risk. In turn this is likely to reflect shorter durations of hospital admission for stratifying clinicians/centres, although this has not been formally examined.

The published evidence presents a strong case that the introduction of less intensive management strategies could offer significant benefits to patients with low-risk FN in terms of reduced hospitalisation and potentially improved quality of life. In a previous UK study, it was estimated that the approximate costs per episode of low-risk FN treated with oral antibiotics/early discharge were $55 \%$ of those for a patient treated as an in-patient treated with i.v. antibiotics (Innes et al, 2003). Approximately $70 \%$ of all FN episodes are low-risk (Klastersky et al, 2000). Thus, if such a management strategy could be safely achieved across the $\mathrm{UK}$, the potential resource savings would be considerable.

There is more than one strategy for using the limited available repertoire of orally bioavailable antibacterials to combat neutropenic infection. Instead of using fluoroquinolones to treat neutropenic infections for patients predicted to be at low risk of complications, they might be used at an earlier time point as prophylaxis. Recent results from a randomised, double-blind, placebo-controlled trial of 1565 patients with solid tumours or lymphoma (Cullen et al, 2005) have shown reduction in the frequency of febrile episodes during chemotherapy in patients receiving levofloxacin prophylaxis compared with those receiving placebo (11 vs $16 \% ; P=0.01)$. These important results are not in themselves sufficient to recommend a universal prophylaxis strategy because the impact on bacterial resistance patterns has not yet been determined. A targeted treatment approach for actual infections using oral fluoroquinolones might enable less intensive treatment and early hospital discharge and these potential benefits need to be assessed. It is not known whether patients who develop FN despite receiving prophylactic antibiotics could be still be defined as 'low-risk' even if they otherwise fulfil the MASCC criteria, since such patients have been excluded from previous studies. Further research in this area is required.

In conclusion, the present study identifies both variations in practice and a degree of caution in implementing evidence-based advances in the management of FN in the UK. Better local and national policies and guidelines are clearly needed. We believe that 
the introduction of newer strategies may be best achieved in the setting of a well-conducted, multicentre trial. The advent of National Cancer Research Networks across the UK provides a unique opportunity for such a study. A randomised phase III trial

\section{REFERENCES}

Bodey B, Groger AM, Bodey Jr B, Siegel SE, Kaiser HE (1997) Immunohistochemical detection of $\mathrm{p} 53$ protein overexpression in primary human osteosarcomas. Anticancer Res 17: 493-498

Cullen M, Steven N, Billingham L, Gaunt C, Hastings M, Simmonds P, Stuart N, Rea D, Bower M, Fernando I, Huddart R, Gollins S, Stanley A (2005) Simple investigation in neutropenic individuals of the frequency of infection after chemotherapy $+/-$ antibiotic in a number of tumours (SIGNIFICANT) trial group. $N$ Engl J Med 353(10): 988-998

Elting LS, Rubenstein EB, Rolston KV, Bodey GP (1997) Outcomes of bacteremia in patients with cancer and neutropenia: observations from two decades of epidemiological and clinical trials. Clin Infect Dis 25: 247-259

Freifeld A, Marchigiani D, Walsh T, Chanock S, Lewis L, Hiemenz J, Hiemenz S, Hicks JE, Gill V, Steinberg SM, Pizzo PA (1999) A doubleblind comparison of empirical oral and intravenous antibiotic therapy for low-risk febrile patients with neutropenia during cancer chemotherapy. $N$ Engl J Med 341: 305-311

Hughes WT, Armstrong D, Bodey GP, Bow EJ, Brown AE, Calandra T, Feld R, Pizzo PA, Rolston KV, Shenep JL, Young LS (2002) 2002 guidelines for the use of antimicrobial agents in neutropenic patients with cancer. Clin Infect Dis 34: 730 -751

Hughes WT, Armstrong D, Bodey GP, Brown AE, Edwards JE, Feld R, Pizzo P, Rolston KV, Shenep JL, Young LS (1997) 1997 guidelines for the use of antimicrobial agents in neutropenic patients with unexplained fever. Infectious Diseases Society of America. Clin Infect Dis 25: 551-573

Hughes WT, Armstrong D, Bodey GP, Feld R, Mandell GL, Meyers JD, Pizzo PA, Schimpff SC, Shenep JL, Wade JC, Young LS, Yow MD (1990) From the Infectious Diseases Society of America. Guidelines for the use of antimicrobial agents in neutropenic patients with unexplained fever. $J$ Infect Dis 161: 381-396

Innes HE, Smith DB, O'Reilly SM, Clark PI, Kelly V, Marshall E (2003) Oral antibiotics with early hospital discharge compared with in-patient intravenous antibiotics for low-risk febrile neutropenia in patients with cancer: a prospective randomised controlled single centre study. $\mathrm{Br} \mathrm{J}$ Cancer 89: $43-49$

Kern WV, Cometta A, De Bock R, Langenaeken J, Paesmans M, Gaya H (1999) Oral versus intravenous empirical antimicrobial therapy for fever comparing standard in-patient management with early hospital discharge in cancer patients receiving oral antibiotics for low-risk FN has been sponsored by Cancer Research UK and will be launched later in the year. in patients with granulocytopenia who are receiving cancer chemotherapy. International Antimicrobial Therapy Cooperative Group of the European Organization for Research and Treatment of Cancer. $N$ Engl Med 341: $312-318$

Klastersky J, Paesmans M, Rubenstein EB, Boyer M, Elting L, Feld R, Gallagher J, Herrstedt J, Rapoport B, Rolston K, Talcott J (2000) The Multinational Association for Supportive Care in Cancer risk index: a multinational scoring system for identifying low-risk febrile neutropenic cancer patients. J Clin Oncol 18: 3038-3051

Paesmans M, Rapoport B, Maertens J, Slabber C, Ferrant A, Wingard J, Aoun M, Dubreucq L, Plehiers B, Klastersky J (2003) Multicentric prospective validation of the mascc risk-index score for identification of febrile neutropeniccancer patients at low-risk for serious medical complications. Proc Am Soc Clin Oncol 22: 556 (abstr 2235)

Rubin M, Hathorn JW, Pizzo PA (1988) Controversies in the management of febrile neutropenic cancer patients. Cancer Invest 6: 167-184

Talcott JA, Rieker P, Clark JA, Propert KJ, Weeks JC, Beard CJ, Wishnow KI, Kaplan I, Loughlin KR, Richie JP, Kantoff PW (1998) Patientreported symptoms after primary therapy for early prostate cancer: results of a prospective cohort study. J Clin Oncol 16: 275-283

Talcott JA, Siegel RD, Finberg R, Goldman L (1992) Risk assessment in cancer patients with fever and neutropenia: a prospective, two-center validation of a prediction rule. J Clin Oncol 10: 316-322

Uys A, Rapoport BL, Anderson R (2004) Febrile neutropenia: a prospective study to validate the Multinational Association of Supportive Care of Cancer (MASCC) risk-index score. Support Care Cancer 12: $555-560$

Vidal L, Paul M, Ben-Dor I, Pokroy E, Soares-Weiser K, Leibovici L (2004) Oral versus intravenous antibiotic treatment for febrile neutropenia in cancer patients. Cochrane Database Syst Rev 18(4): CD003992

Viscoli C, Bruzzi P, Castagnola E, Boni L, Calandra T, Gaya H, Meunier F, Feld R, Zinner S, Klastersky J, Glauser M (1994) Factors associated with bacteraemia in febrile, granulocytopenic cancer patients. The International Antimicrobial Therapy Cooperative Group (IATCG) of the European Organization for Research and Treatment of Cancer (EORTC). Eur J Cancer 30A: 430-437 